\title{
Study of nonlinear ion- and electron-acoustic waves in multi-component space plasmas
}

\author{
G. S. Lakhina ${ }^{1}$, S. V. Singh ${ }^{1}$, A. P. Kakad ${ }^{1}$, F. Verheest ${ }^{2,3}$, and R. Bharuthram ${ }^{4}$ \\ ${ }^{1}$ Indian Institute of Geomagnetism, New Panvel (W), Navi Mumbai - 410 218, India \\ ${ }^{2}$ Sterrenkundig Observatorium, Universiteit Gent, Krijgslaan 281, B-9000 Gent, Belgium \\ ${ }^{3}$ School of Physics, University of KwaZulu-Natal, Private Bag X54001, Durban 4000, South Africa \\ ${ }^{4}$ University of the Western Cape, Modderdam Road, Belville 7530, South Africa
}

Received: 30 June 2008 - Revised: 25 September 2008 - Accepted: 2 October 2008 - Published: 27 November 2008

\begin{abstract}
Large amplitude ion-acoustic and electronacoustic waves in an unmagnetized multi-component plasma system consisting of cold background electrons and ions, a hot electron beam and a hot ion beam are studied using Sagdeev pseudo-potential technique. Three types of solitary waves, namely, slow ion-acoustic, ion-acoustic and electron-acoustic solitons are found provided the Mach numbers exceed the critical values. The slow ion-acoustic solitons have the smallest critical Mach numbers, whereas the electron-acoustic solitons have the largest critical Mach numbers. For the plasma parameters considered here, both type of ion-acoustic solitons have positive potential whereas the electron-acoustic solitons can have either positive or negative potential depending on the fractional number density of the cold electrons relative to that of the ions (or total electrons) number density. For a fixed Mach number, increases in the beam speeds of either hot electrons or hot ions can lead to reduction in the amplitudes of the ion-and electron-acoustic solitons. However, the presence of hot electron and hot ion beams have no effect on the amplitudes of slow ion-acoustic modes. Possible application of this model to the electrostatic solitary waves (ESWs) observed in the plasma sheet boundary layer is discussed.
\end{abstract}

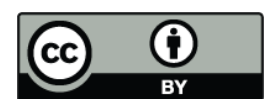

Correspondence to: G. S. Lakhina (lakhina@iigs.iigm.res.in)

\section{Introduction}

The boundary layers of the Earth's magnetosphere can support a variety of electrostatic and electromagnetic wave modes, e.g., Alfvén modes, whistler waves, broadband electrostatic noise (BEN), etc. Analysis of the high time resolution of the plasma wave data from GEOTAIL have shown that BEN in the plasma sheet boundary layer actually consists of short electrostatic solitary waves (ESWs) whose Fourier spectrum give rises to the broadband nature of the noise (Matsumoto et al., 1994). ESWs have been observed at/in the bow shock (Bale et al., 1998), the magnetosheath (Pickett et al., 2003), the polar cap boundary layer (Franz et al., 1998; Tsurutani et al., 1998), and in the auroral acceleration region (Ergun et al., 1998; Bounds et al, 1999). It is interesting to note that the electrostatic solitary structures are observed in the electric field parallel to the background magnetic field, are usually bipolar or tripolar, and their amplitudes are typically a few $\mathrm{mV} / \mathrm{m}$ in the plasma sheet boundary layer, but they can be as large as $200 \mathrm{mV} / \mathrm{m}$ at polar altitudes (Cattell et al., 1999).

The ESWs are generally associated with electron or/and ion beams. The ESWs associated with the ion beam regions of the auroral zone usually have negative potentials and propagate at velocities of the order of ion-acoustic or beam speed (Temerin et al., 1982; Boström et al., 1988; Koskinen et al., 1990). The negative potential ESWs have been interpreted in terms of ion solitary waves or solitons (Koskinen et al., 1990; Hudson et al., 1983; Reddy and Lakhina, 1991; Reddy et al., 1992). In other regions of the Earth's magnetosphere, the ESWs have usually positive potentials, and they propagate at velocities comparable to the electron thermal velocity $\left(\sim 1000 \mathrm{~s}\right.$ of $\left.\mathrm{km} \mathrm{s}^{-1}\right)$. The positive potential ESWs have

Published by Copernicus Publications on behalf of the European Geosciences Union and the American Geophysical Union. 
been commonly interpreted in terms of as Bernstein-GreeneKruskal (BGK) modes (Bernstein et al., 1957; Muschietti et al., 1999; Jovanovic and Shukla, 2000; Chen et al., 2005). Other favorite interpretations for these ESWs are based on the nonlinear evolution of a bump-on-tail instability/electron two stream instability (Omura et al., 1996; Kojima et al., 1997; Goldman et al., 1999), and electron-acoustic solitary waves (Pottelette et al., 1990; Dubouloz et al., 1991, 1993; Singh et al., 2001; Singh and Lakhina, 2001, 2004; Tagare et al., 2004, Lakhina et al., 2008). For a detailed discussion of various models, one can refer to Lakhina et al. $(2000,2004)$.

It is well known that multi-species plasma, e.g., cold background (electron-ion) plasma and cold/hot electron-ion beams can exist in the magnetosphere. The cold plasma (temperatures a few eVs) originates in the ionosphere and the hot plasma (temperatures $100 \mathrm{~s} \mathrm{eVs}$ to a few $\mathrm{keVs}$ ) comes from the magnetosphere. The ring current region has plasmas having energies of several $100 \mathrm{keV}$ to several MeV. The intermixing of cold and hot plasmas occurs in the flow boundary, like, magnetopause, plasma sheet boundary layer, polar cusp and auroral field lines, etc. The multi-component plasmas containing cold and hot electrons and ions, formed by the mixing of two plasmas of different temperatures could exist on a time scale shorter than the thermalization time, which in the collisionless space plasmas could be very large, of the order of several hours to days in the magnetosphere. Several spacecrafts have indeed observed multispecies plasmas in the magnetosphere (Tokar and Gary, 1984; Lin et al., 1984; Koskinen et al, 1990; Onsager et al., 1993; Matsumoto et al., GRL, 1994; Pottelette et al., 1999; Vogiatzis et al., 2006; Teste et al., 2007; Backrud-Ivgren et al., 2005; Pickett et al., 2005).

There is a vast body of theoretical literature employing multi-species plasma models to study ion-acoustic and electron-acoustic waves. The nonlinear ion-acoustic waves in multi-species plasmas have been studied by several workers (Buti, 1980; Hudson et al., 1983; Qian et al., 1988; Bharutram and Shukla, 1986; Berthomier et al., 1998; Reddy and Lakhina, 1991; Reddy et al., 1992). The electronacoustic waves in multi-species plasmas have been discussed by Watanabe and Tanuti (1977); Gary and Tokar (1985); Mace and Hellberg (1985, 2001); Ashour-Abdalla and Okuda (1986); Pottelette et al. (1990); Dubouloz et al. (1991, 1993); Berthomier et al. (2000, 2003); Singh et al. (2001); Tagare et al. (2004); Cattaert et al. (2005), Kakad et al. (2007) and Lakhina et al. (2008).

The earlier models of electron-acoustic solitons considered two/three temperature electron plasmas, and they could explain the space observations (e.g. Viking) of solitary waves which had negative potentials (Pottelette et al., 1990; Dubouloz et al., 1991, 1993; Singh et al., 2001; Singh and Lakhina, 2004; Tagare et al., 2004). To explain the positive potential structures, Berthomier et al. (2000) introduced an electron beam in the system having cold and hot electrons. Later on, electron-acoustic solitons in such three-electron (cold, hot, beam) component plasma system were studied by Mace and Hellberg (2001), Berthomier et al. (2003) and ElTaibany (2004). These models show that depending on the beam density and temperature and below a critical velocity of the electron beam, nonlinear structures can have a positive potential signature. Recently, Verheest et al. (2005) have pointed out the possibility to obtain compressive electronacoustic solitons, those having positive potentials, even without the electron-beam component, provided the hot electron inertia is retained in the analysis. The coexistence of rarefactive and compressive electron-acoustic solitary waves for some specific plasma parameters has been predicted in a four-component unmagnetized plasma system consisting of cold background electrons, a cold electron beam, and two types of ion species, i.e., cold and hot ions having Boltzmann distributions (Kakad et al., 2007). More recently, Ghosh et al. (2008) have studied electron-acoustic solitary waves in a four-component plasma consisting of warm electrons, warm electron beam and two types of hot ions, and found that the ions temperature and the concentration control the characteristics and the existence domain of the positive potential solitons.

Recently, Lakhina et al. (2008) have studied a threecomponent plasma system consisting of cold and hot electrons and one type of ions. Solitary wave solutions were found when the Mach numbers exceed some critical values. The critical Mach numbers for the ion-acoustic solitons are found to be smaller than those for electron-acoustic solitons for a given set of plasma parameters. The ion-acoustic solitons had positive potentials for the parameters considered. However, the electron-acoustic solitons could have either positive or negative potentials depending on whether the fractional cold electron density with respect to the ion density was greater or less than a certain critical value. In this paper, we extend the work of Lakhina et al. (2008) by introducing second ion species, i.e., a hot ion beam in the system. In addition, the hot electrons will also have a beam component. We develop a general formalism employing a multi-fluid approach. Thus, the restrictive assumption of treating the hot electrons and/or hot ions as having Boltzmann distributions considered in many earlier studies (e.g., Singh et al., 2001; Kakad et al., 2007) is removed in the present analysis.

\section{Model}

We consider an infinite, collisionless and unmagnetized plasma consisting of four components, namely, cold electrons ( $\left.N_{\text {ce }}, T_{\text {ce }}\right)$, hot beam electrons $\left(N_{\text {he }}, T_{\text {he }}, v_{\text {he }}\right)$, cold ions $\left(N_{\mathrm{ci}}, T_{\mathrm{ci}}\right)$, and hot beam ions $\left(N_{\mathrm{hi}}, T_{\mathrm{hi}}, v_{\mathrm{hi}}\right)$, where $N_{j}, T_{j}$, $v_{j}$ represents the equilibrium density, temperature and beam velocity (along the direction of wave propagation) of the species $j$, and $j=c e$, he, ci and hi for the cold electrons, hot electrons, cold ions and the hot ions, respectively. We treat all the species as mobile. Then, their dynamics is governed 
by the multi-fluid equations of continuity, momentum, and equation of state of each species, and the Poisson equation (Lakhina et al., 2008).

$$
\begin{aligned}
& \frac{\partial n_{j}}{\partial t}+\frac{\partial\left(n_{j} v_{j}\right)}{\partial x}=0 \\
& \frac{\partial v_{j}}{\partial t}+v_{j} \frac{\partial v_{j}}{\partial x}+\frac{1}{\mu_{j} n_{j}} \frac{\partial P_{j}}{\partial x}-\frac{Z_{j}}{\mu_{j}} \frac{\partial \phi}{\partial x}=0 \\
& \frac{\partial P_{j}}{\partial t}+v_{j} \frac{\partial P_{j}}{\partial x}+3 P_{j} \frac{\partial v_{j}}{\partial x}=0 \\
& \frac{\partial^{2} \phi}{\partial x^{2}}=n_{\mathrm{ce}}+n_{\mathrm{he}}-n_{\mathrm{ci}}-n_{\mathrm{hi}}
\end{aligned}
$$

Here, $\mu_{j}=m_{j} / m_{i}$, and $Z_{j}$ is the electronic charge of the $j$ th species, i.e, $Z_{\mathrm{ce}, \mathrm{he}}=-e$, and $Z_{\mathrm{ci}, \mathrm{hi}}=l_{\mathrm{ci}, \mathrm{hi}} e$, where $l_{c i, h i}$ is the number denoting the ionization state of the ions, e.g., $l_{\mathrm{ci}}=l_{\mathrm{hi}}=1$ for protons. Further, all densities are normalized with the total unperturbed ion or electron density, $N_{0}=N_{\text {ci }}+N_{\text {hi }}=N_{\text {ce }}+N_{\text {he }}$, velocities with the hot ion thermal velocity $C_{\mathrm{hi}}=\left(T_{\mathrm{hi}} / m_{i}\right)^{1 / 2}$ (here, $m_{j}$ represent the mass of the $j$ th species), time with the with inverse of ion plasma frequency, $\omega_{p i}^{-1}$, where $\omega_{p i}=\sqrt{4 \pi N_{0} e^{2} / m_{i}}$, the lengths with the ion Debye length, $\lambda_{d i}=\sqrt{T_{\mathrm{hi}} / 4 \pi N_{0} e^{2}}$, potential $\phi$ with $T_{\mathrm{hi}} / e$, and the thermal pressures $P_{j}$ with $N_{0} T_{\mathrm{hi}}$. Furthermore, we have assumed the same adiabatic index, i.e., $\gamma=3$, for all the species in the equation of state given by Eq. (3).

To study the properties of stationary arbitrary amplitude ESWs, we transform the above set of equations to a stationary frame moving with velocity $v$, the phase velocity of the wave, i.e., $\xi=(x-M t)$, where $M=V / C_{\mathrm{hi}}$ is the Mach number with respect to the hot ion thermal velocity. Then, solving for perturbed densities, putting these expressions in the Poisson equation, and assuming appropriate boundary conditions for the localized disturbances along with the conditions that $\phi=0$, and $d \phi / d \xi=0$ at $\xi \rightarrow \pm \infty$, we get the following energy integral,

$\frac{1}{2}\left(\frac{\partial \phi}{\partial \xi}\right)^{2}+\psi(\phi, M)=0$

where

$$
\begin{aligned}
\psi(\phi, M) & =\mu_{e} n_{\mathrm{ce}}^{0}\left\{M^{2}-\frac{M}{\sqrt{2}} B_{\mathrm{ce}}^{1 / 2}\right\} \\
& +n_{\mathrm{ce}}^{0} T_{\mathrm{ce}}\left\{1-2 \sqrt{2} M^{3} B_{\mathrm{ce}}^{-3 / 2}\right\} \\
& +\mu_{e} n_{\mathrm{he}}^{0}\left\{\left(M-\mathrm{v}_{\mathrm{he}}\right)^{2}-\frac{\left(M-\mathrm{v}_{\mathrm{he}}\right)}{\sqrt{2}} B_{\mathrm{he}}^{1 / 2}\right\} \\
& +n_{\mathrm{he}}^{0} T_{\mathrm{he}}\left\{1-2 \sqrt{2}\left(M-\mathrm{v}_{\mathrm{he}}\right)^{3} B_{\mathrm{he}}^{-3 / 2}\right\} \\
& +n_{\mathrm{ci}}^{0}\left\{M^{2}-\frac{M}{\sqrt{2}} B_{\mathrm{ci}}^{1 / 2}\right\}+n_{\mathrm{ci}}^{0} T_{\mathrm{ci}}\left\{1-2 \sqrt{2} M^{3} B_{\mathrm{ci}}^{-3 / 2}\right\} \\
& +n_{\mathrm{hi}}^{0}\left\{\left(M-\mathrm{v}_{\mathrm{hi}}\right)^{2}-\frac{\left(M-\mathrm{v}_{\mathrm{hi}}\right)}{\sqrt{2}} B_{\mathrm{hi}}^{1 / 2}\right\} \\
& +n_{\mathrm{hi}}^{0}\left\{1-2 \sqrt{2}\left(M-\mathrm{v}_{\mathrm{hi}}\right)^{3} B_{\mathrm{hi}}^{-3 / 2}\right\}
\end{aligned}
$$

is the pseudopotential, also known as the Sagdeev potential. Here,

$$
\begin{aligned}
& B_{\mathrm{ce}}=A_{\mathrm{ce}}+\sqrt{A_{\mathrm{ce}}^{2}-\frac{12 T_{\mathrm{ce}} M^{2}}{\mu_{e}}}, \\
& B_{\mathrm{he}}=A_{\mathrm{he}}+\sqrt{A_{\mathrm{he}}^{2}-\frac{12 T_{\mathrm{he}}\left(M-\mathrm{v}_{\mathrm{he}}\right)^{2}}{\mu_{e}}}, \\
& B_{\mathrm{ci}}=A_{\mathrm{ci}}+\sqrt{A_{\mathrm{ci}}^{2}-12 T_{\mathrm{ci}} M^{2}}, \\
& B_{\mathrm{hi}}=A_{\mathrm{hi}}+\sqrt{A_{\mathrm{hi}}^{2}-12\left(M-\mathrm{v}_{\mathrm{hi}}\right)^{2}}, \\
& A_{\mathrm{ce}}=M^{2}+\frac{3 T_{\mathrm{ce}}}{\mu_{e}}+\frac{2 \phi}{\mu_{e}}, \\
& A_{\mathrm{he}}=\left(M-\mathrm{v}_{\mathrm{he}}\right)^{2}+\frac{3 T_{\mathrm{he}}}{\mu_{e}}+\frac{2 \phi}{\mu_{e}}, \\
& A_{\mathrm{ci}}=M^{2}+3 T_{\mathrm{ce}}-2 \phi, A_{\mathrm{hi}}=\left(M-\mathrm{v}_{\mathrm{hi}}\right)^{2}+3-2 \phi, \\
& \mu_{e}=\frac{m_{e}}{m_{i}} .
\end{aligned}
$$

Further, in Eq. (6), $n_{j}^{0}=N_{j} / N_{i}$ such that $n_{\mathrm{ce}}^{0}+n_{\mathrm{he}}^{0}=n_{\mathrm{ci}}^{0}+n_{\mathrm{hi}}^{0}=1$, and the temperatures of the species are normalized with the hot ion temperature. On taking $n_{\mathrm{ci}}^{0}=0$, Eq. (6) reduces to that derived by Lakhina et al. (2008).

\section{Nonlinear Electrostatic Solitary Waves (ESWs)}

Equation (5) yields solitary wave solutions when the Sagdeev potential satisfies the following conditions: $\psi(\phi, M)=0$, $d \psi(\phi, M) / d \phi=0, d^{2} \psi(\phi, M) / d \phi^{2}<0$ at $\phi=0 ; \psi(\phi, M)=0$ at $\phi=\phi_{0}$, and $\psi(\phi, M)<0$ for $0<|\phi|<\left|\phi_{0}\right|$. From Eq. (6) it is seen that $\psi(\phi, M)$ and its first derivative with respect to $\phi$ vanish at $\phi=0$. The condition $d^{2} \psi(\phi, M) / d \phi^{2}<0$ at $\phi=0$ is satisfied provided $M>M_{0}$, where $M_{0}$ satisfies the equation

$$
\begin{aligned}
f\left(M_{0}\right) & \equiv \frac{n_{\mathrm{ce}}^{0}}{\mu_{e}\left[M_{0}^{2}-\frac{3 T_{\mathrm{ce}}}{\mu_{e}}\right]}+\frac{n_{\mathrm{he}}^{0}}{\mu_{e}\left[\left(M_{0}-\mathrm{v}_{\mathrm{he}}\right)^{2}-\frac{3 T_{\mathrm{he}}}{\mu_{e}}\right]} \\
& +\frac{n_{\mathrm{ci}}^{0}}{\left[M_{0}^{2}-3 T c i\right]}+\frac{n_{\mathrm{hi}}^{0}}{\left[\left(M_{0}-\mathrm{v}_{\mathrm{hi}}\right)^{2}-3\right]}=0
\end{aligned}
$$

Equation (7) yields 6 roots but all the roots will not be physical. We will consider here only the real positive roots, or the critical Mach numbers. Numerical solution of Eq. (7) shows the existence of three critical Mach numbers which satisfy all the soliton conditions. There is no physical root below the cold ion thermal velocity. The relative ordering of the thermal velocities of the species decides where the physical roots lie. For example, the lowest root lie between the cold ion thermal velocity, $C_{\mathrm{ci}}$, and the smaller of the hot ion $\left(C_{\mathrm{hi}}\right)$ or cold electron $\left(C_{\mathrm{ce}}\right)$ thermal velocities. The 

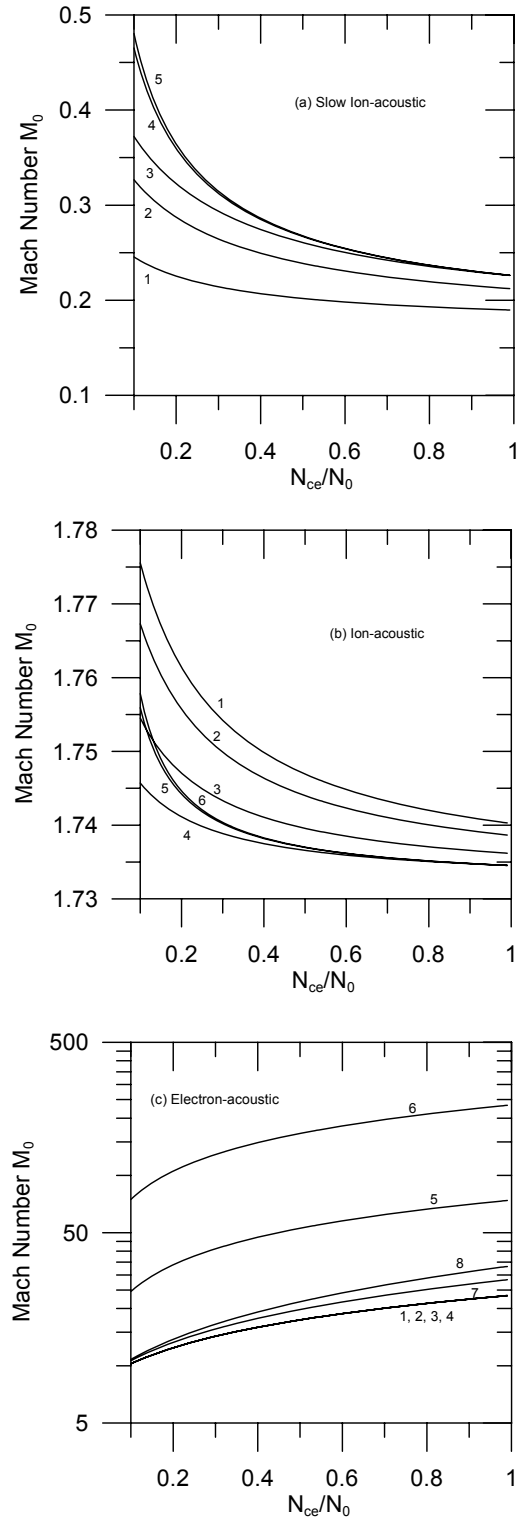

Fig. 1. Variation of critical Mach number $M_{0}$ versus cold electron to total electron density ratio $N_{\mathrm{ce}} / N_{0}$ for the parameters: $T_{\mathrm{ce}} / T_{\mathrm{hi}}=0.01, T_{\mathrm{ci}} / T_{\mathrm{hi}}=0.01, v_{\mathrm{he}}=0, v_{\mathrm{hi}}=0$. Panel a shows slow ionacoustic critical mach numbers. For curves 1,2 and $3, T_{\mathrm{he}} / T_{\mathrm{hi}}=0.1$ and $N_{\mathrm{ci}} / N_{0}=0.2,0.5$ and 0.7 , respectively. For curves 4 and 5, $N_{\text {ci }} / N_{0}=0.7$ and $T_{\text {he }} / T_{\text {hi }}=1.0$ and 10.0 , respectively. Panel b shows ion-acoustic mode critical mach numbers. For curves 1, 2, 3 and $4, T_{\mathrm{he}} / T_{\mathrm{hi}}=0.1$ and $N_{\mathrm{ci}} / N_{0}=0.0,0.2,0.5$ and 0.7 , respectively. For curves 5 and $6, N_{\mathrm{ci}} / N_{0}=0.7$ and $T_{\mathrm{he}} / T_{\mathrm{hi}}=1.0$ and 10.0 , respectively. Panel c shows electron-acoustic Mach numbers for the same parameters for curves $1-6$ as in Panel b. For the curves 7 and $8, N_{\mathrm{ci}} / N_{0}=0.7, T_{\mathrm{he}} / T_{\mathrm{hi}}=0.1$, and $v_{\mathrm{he}}=5.0$ and 10.0 , respectively. Variation in cold ion density ratio $N_{\text {ci }} / N_{0}$ has no effect on the electron-acoustic critical Mach number (cf. curves 1, 2, 3 and 4). second root (intermediate) lies either between $C_{\mathrm{hi}}$ and $C_{\mathrm{ce}}$ if $C_{\mathrm{hi}}<C_{\mathrm{ce}}$ or between $C_{\mathrm{ce}}$ and $C_{\mathrm{hi}}$ if $C_{\mathrm{hi}}>C_{\mathrm{ce}}$. The third (highest) root lies between the greater of $C_{\mathrm{hi}}$ and $C_{\mathrm{ce}}$ and the hot electron thermal velocity, $C_{\text {he }}$. It is interesting to note that in a three-component plasma system consisting of cold and hot electrons and ions, Lakhina et al. (2008) obtained two modes corresponding to the intermediate and highest roots described above. They called these modes as ion-acoustic and electron acoustic, respectively (see Verheest et al., 2007). The introduction of the second cold ion species in the model by Lakhina et al. (2008) results in the third (lowest) root. Since the phase velocity of the new mode is greater than the ion-acoustic speed with respect to cold electron temperature, i.e., $M>\left(T_{\mathrm{ce}} / m_{i}\right)^{1 / 2} / C_{\mathrm{hi}}=\left(T_{\mathrm{ce}} / T_{\mathrm{hi}}\right)^{1 / 2}$, we tentatively call this new mode as the slow ion-acoustic to distinguish it from the other two modes. The intermediate and the highest roots will be called as ion-acoustic and electron-acoustic, respectively.

Figure 1 shows the variations of the roots of Eq. (7), i.e, the critical Mach numbers, versus the ratio of cold electron to total electron number density, $N_{\mathrm{ce}} / N_{0}$, for different values of $N_{\mathrm{ci}} / N_{0}$ and $T_{\mathrm{he}} / T_{\mathrm{hi}}$. Panels a, b and c show the critical Mach numbers for the slow ion-acoustic, ion- acoustic and the electron-acoustic modes, respectively. Further, in a fluid dynamic formalism, when inertia for all species is retained (as done here), the respective Mach numbers are limited by sonic points, where the flow of one species is choked (McKenzie et al., 2004; Verheest et al., 2004). The critical Mach numbers correspond to this situation.

From panel a of Fig. 1, it seen that the critical Mach numbers for the slow ion-acoustic modes show decrease with the increase in cold electron density ratio $N_{\mathrm{ce}} / N_{0}$, but they show increase with the increase in cold ion density ratio $N_{\text {ci }} / N_{0}$ (cf. curves 1,2 and 3). The critical Mach number show a slight increase when the temperature ratio $T_{\mathrm{he}} / T_{\mathrm{hi}}$ increases from 1 to 10 (see curves 4 and 5). From Panel b of Fig. 1, it is clear that the critical Mach number for the ion acoustic modes are decreased with the increase of $N_{\text {ce }} / N_{0}$ as well as $N_{\text {ci }} / N_{0}$ (cf. curves $1,2,3,4$ ). The variation of $T_{\text {he }} / T_{\text {hi }}$ from 1 to 10 results in a slight increase in the critical Mach numbers (cf. curves 5 and 6). Panel c of Fig. 1 shows that the critical Mach numbers for the electron-acoustic modes are increased by an increase in cold electron density ratio $N_{\text {ce }} / N_{0}$ (all curves), hot electron temperature ratio $T_{\text {he }} / T_{\text {hi }}$ (curves 4,5 and 6) and hot electron beam velocity (cf. curves 4, 7 and 8). The increase in cold ion density has no effect on the critical Mach numbers (cf. curves 1, 2, 3 and 4).

We have numerically solved Eq. (6) for the Sagdeev potential, $\psi(\phi, M)$, as a function of $\phi$ for various values of Mach numbers and for some typical plasma parameters. The results are shown in Figs. 2 and 3. The solitary wave solutions for all three modes, e.g., slow ion-acoustic, ion- and electronacoustic modes, are found when the Mach numbers exceed the critical values (e.g., as found in Fig. 1). 
In Fig. 2, it is seen that both the slow ion-acoustic (panel a) and the ion-acoustic (panel b) solitons have positive potentials. The Mach numbers needed for the soliton solutions are smaller for the slow ion-acoustic mode than that of ionacoustic mode. The maximum electrostatic potential $\phi_{0}$ increases with the increase of the Mach number, $M$, as seen from the curves $1,2,3$ and 4 of panel a and b. In both the panels, the soliton solution does not exist for the curve 5. Hence there is an upper value for $M$, say $M_{\max }$, above which soliton solutions do not exist. This is also true for the electron-acoustic solitary structures (curve 5 of panel c). Panel c shows that for the same plasma parameters, but for still higher values of the Mach number, $M$, electron-acoustic solitons can exist. However, the electron-acoustic solitons have negative potentials. In this case also the maximum electrostatic potential $\phi_{0}$ increases with an increase in $M$.

Figure 3 shows the variation of $\psi(\phi, M)$ versus $\phi$ for the same plasma parameters as in Fig. 2 except for $N_{\text {ce }} / N_{i}$. Here $N_{\text {ce }} / N_{i}=0.9$ whereas it is 0.3 in Fig. 2 . Here, all the three modes, namely, slow ion-acoustic (panel a), ion-acoustic (panel b) and electron-acoustic (panel c) solitons, have positive potentials. For all the three types of solitons, $\phi_{0}$ increases with $M$, and the solution do not exist for Mach numbers corresponding to curve 5 or higher values.

For the parameters considered for the computations, the model supports only positive potential slow ion-acoustic and ion-acoustic solitons. However, it can support either positive or negative potential electron-acoustic solitons depending on the fractional cold electron density, i.e., the ratio $R=N_{\text {ce }} / N_{0}$. Starting from small values of $R$, a change over from negative to positive potential structures occurs when $R$ exceed a certain critical value, which depends on the plasma parameters. For example, for the case of $T_{\mathrm{ce}} / T_{\mathrm{hi}}=0.01, T_{\mathrm{he}} / T_{\mathrm{hi}}=1.0$ and no beams, the electron-acoustic solitons have negative potentials for $R<0.43$ and positive potentials when $R \geq 0.43$.

In Fig. 4, we have shown $\phi_{\max }$, the maximum value of $\phi_{0}$ corresponding to $M_{\max }$ (beyond which solitary solutions do not exist) for different values of the hot electron to ion temperature ratio, $T_{\text {he }} / T_{\text {hi }}$. From panel a and b of Fig. 4 , it is seen that the slow ion-acoustic and ion-acoustic solitons have positive potentials which first increase with $T_{\mathrm{he}} / T_{\mathrm{hi}}$ and then saturate. The electron-acoustic solitons have negative potential for $N_{\text {ce }} / N_{0}=0.3$ (cf. curve 1, panel c) and positive potential for $N_{\mathrm{ce}} / N_{0}=0.9$ (curve 2 , panel c), and the magnitude of $\phi_{\max }$ increases monotonically with an increase of $T_{\text {he }} / T_{\text {hi }}$. Similar behavior is also seen at other densities. The magnitude of $\phi_{\max }$ decreases as $N_{\text {ce }} / N_{0}$ increases (cf. curves 1 and 2 in all panels). The increase of cold electron to hot ion temperature ratio $T_{\mathrm{ce}} / T_{\mathrm{hi}}$ leads to increase of $\phi_{\max }$ for slow ion-acoustic and ion-acoustic modes (cf. curves 1 and 3 of panels a and b) but a slight decrease in electron acoustic (cf. curve 1 and 3 of panel c) solitary structures. However, at higher cold electron densities, the electron-acoustic solitons have positive potentials, and the $\phi_{\max }$ magnitude once again increases monotonically with $T_{\mathrm{he}} / T_{\mathrm{hi}}$. Further, an increase
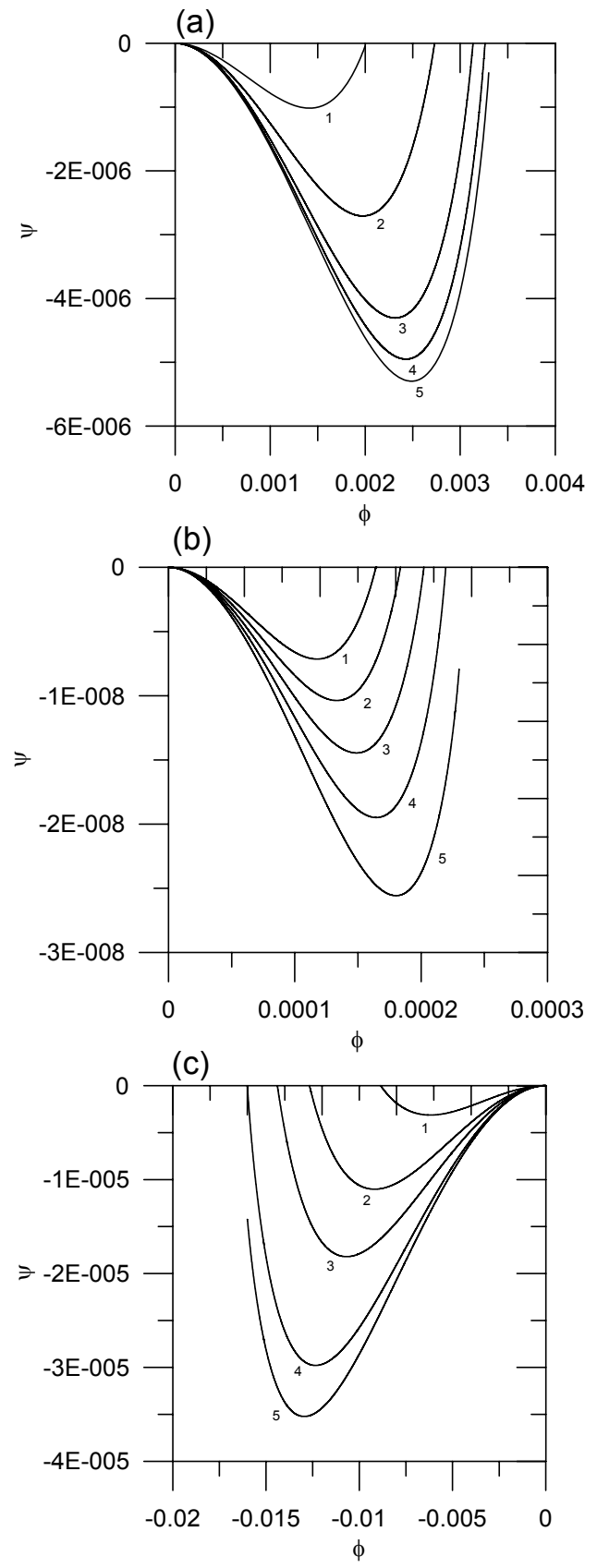

Fig. 2. Slow ion-acoustic (panel a), ion acoustic (panel b) and electron acoustic (panel c) solitons for plasma parameters: $N_{\text {ce }} / N_{0}=0.3, N_{\text {ci }} / N_{0}=0.3, T_{\text {ce }} / T_{\mathrm{hi}}=0.01, T_{\text {ci }} / T_{\mathrm{hi}}=0.01, v_{\mathrm{he}}=0$, $v_{\mathrm{hi}}=0$, and $T_{\mathrm{he}} / T_{\mathrm{hi}}=0.1$. For panel a, the Mach numbers $M=0.2450$, $0.2500,0.2530,0.2540$ and 0.2545 for curves $1,2,3,4$ and 5 , respectively. For panel b, $M=1.7515,1.7520,1.7525,1.7530$ and 1.7535 for curves 1, 2, 3, 4 and 5, respectively. For panel c, $M=14.70,14.90,15.00,15.11$ and 15.15 for curves $1,2,3,4$ and 5 , respectively. In all panels, the soliton solutions do not exist for Mach numbers corresponding to curve 5 or higher values. 
(a)

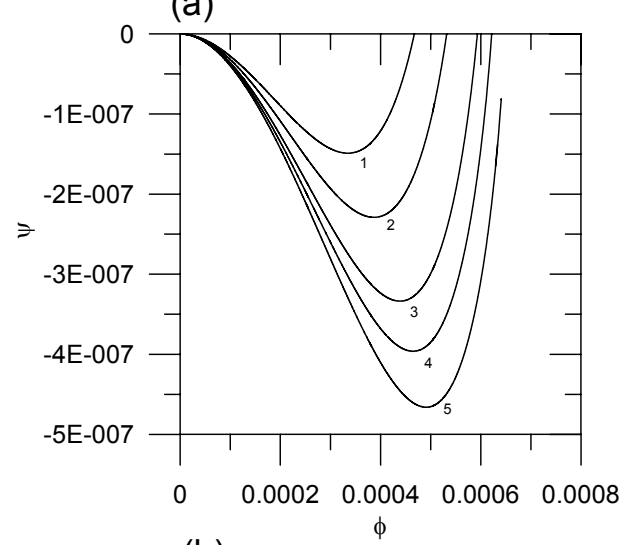

(b)

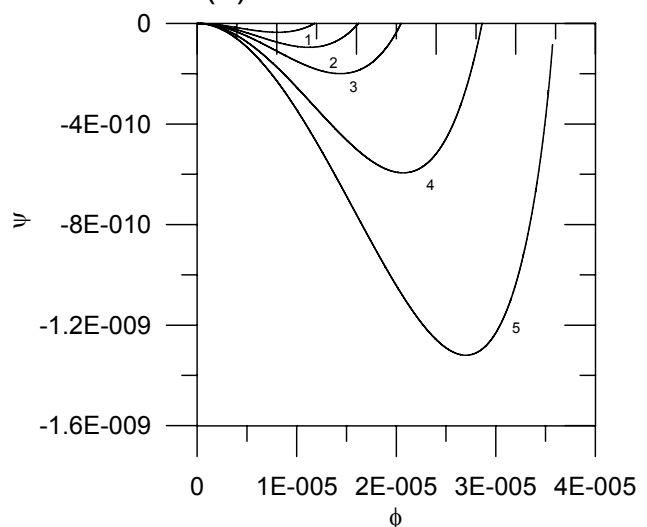

(c)

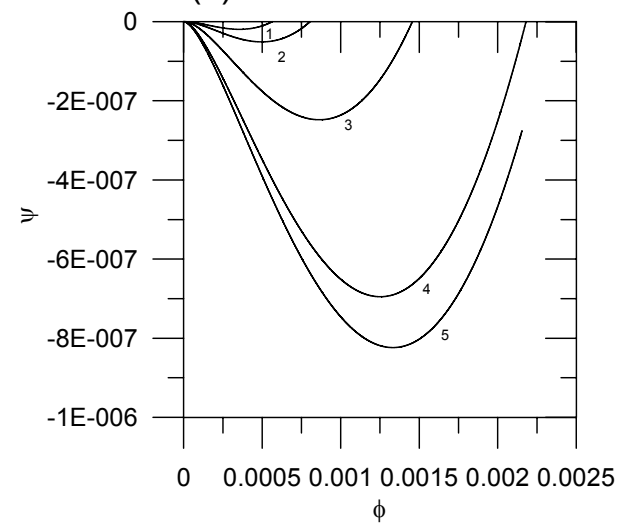

Fig. 3. Slow ion-acoustic (panel a), ion acoustic (panel b) and electron acoustic (panel c) solitons for plasma for same plasma parameters as Fig. 2 except that $N_{\mathrm{ce}} / N_{0}=0.9$. For panel a, the Mach numbers $M=0.2060,0.2070,0.2080,0.2085$ and 0.2090 for curves 1,2 , 3, 4 and 5, respectively. For panel b, $M=1.7390,1.7392,1.7395$, 1.7400 and 1.7405 for curves 1, 2, 3, 4 and 5, respectively. For panel c, $M=22.65,22.75,23.00,23.25$ and 23.30 for curves 1, 2, 3, 4 and 5, respectively. In all panels, the soliton solutions do not exist for Mach numbers corresponding to curve 5 or higher values. (a)

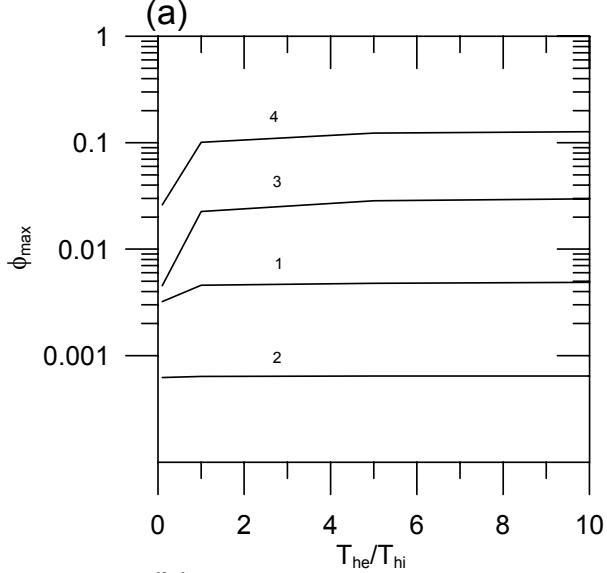

(b)

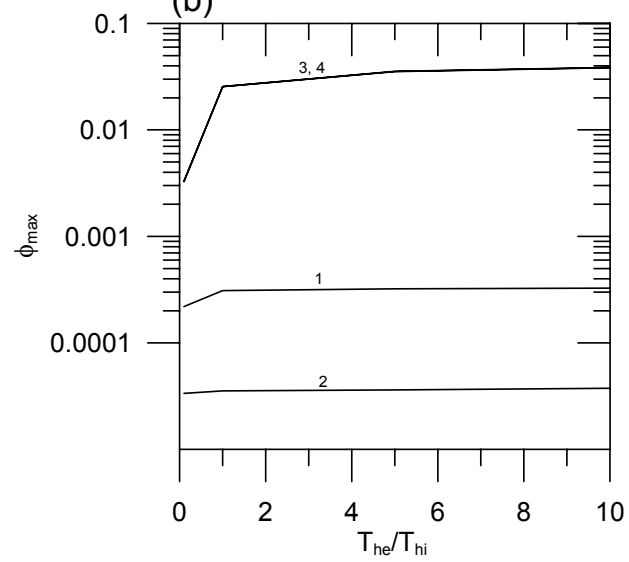

(c)

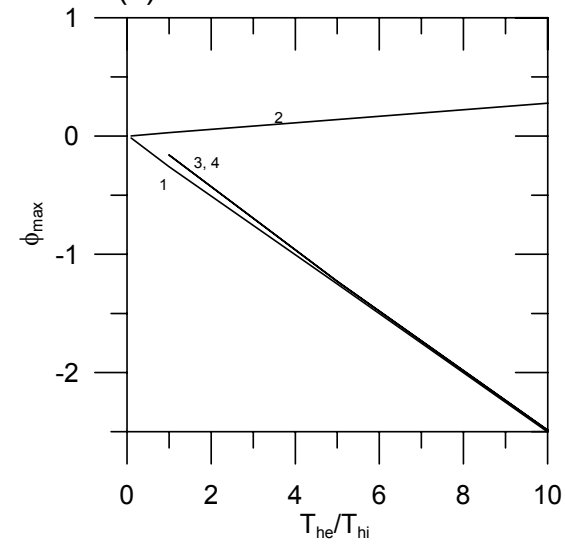

Fig. 4. Variation of $\phi_{\max }$, the maximum value of $\phi_{0}$ corresponding to $M_{\max }$ (beyond which solitary solutions do not exist), versus hot electron to ion temperature ratio, $T_{\mathrm{he}} / T_{\mathrm{hi}}$ for the slow ion-acoustic (panel a), ion-acoustic (panel b) and electronacoustic (panel c) solitons for plasma parameters: $N_{\mathrm{ci}} / N_{0}=0.3$, $T_{\mathrm{ce}} / T_{\mathrm{hi}}=0.01, T_{\mathrm{ci}} / T_{\mathrm{hi}}=0.01, v_{\mathrm{he}}=0, v_{\mathrm{hi}}=0$. For curves 1 and 2 , $N_{\text {ce }} / N_{0}=0.3$ and 0.9 . For curve $3, N_{\text {ce }} / N_{0}=0.3, T_{\text {ce }} / T_{\mathrm{hi}}=0.1$ and $T_{\mathrm{ci}} / T_{\mathrm{hi}}=0.01$. For curve $4, N_{\mathrm{ce}} / N_{0}=0.3, T_{\mathrm{ce}} / T_{\mathrm{hi}}=0.1$ and $T_{\mathrm{ci}} / T_{\mathrm{hi}}=0.1$. 


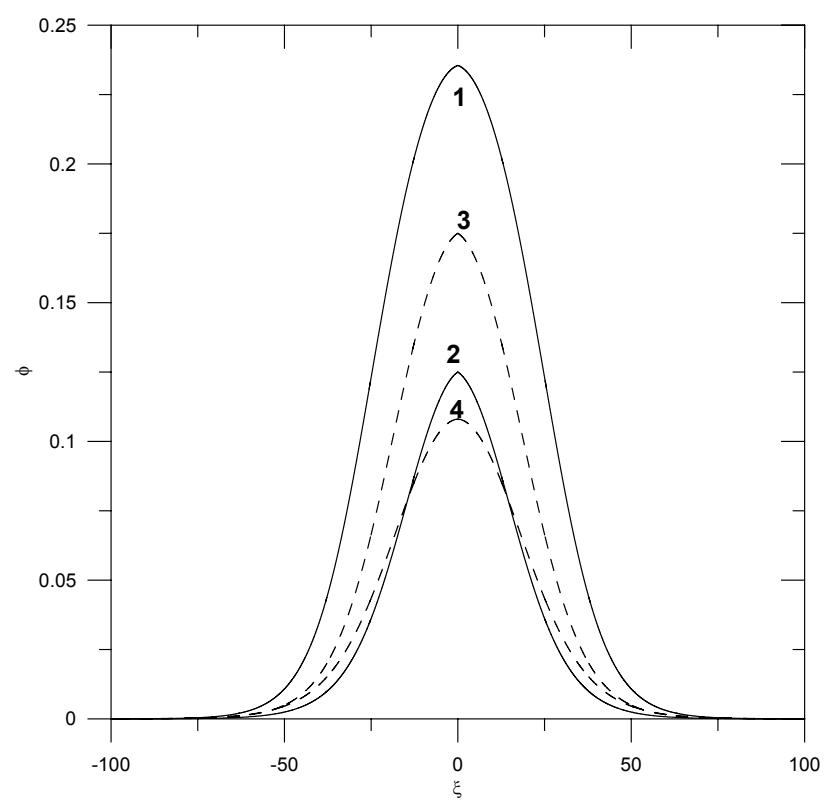

Fig. 5. Shows variation of the electron-acoustic potential $\phi$ versus $\xi$ for $N_{\mathrm{ce}} / N_{0}=0.5, N_{\mathrm{ci}} / N_{0}=0.3, T_{\mathrm{ce}} / T_{\mathrm{hi}}=T_{\mathrm{ci}} / T_{\mathrm{hi}}=0.01$, $T_{\mathrm{he}} / T_{\mathrm{hi}}=1.0$, and $M=53.429$. For solid curves, $v_{\mathrm{he}}=0$, and $v_{\mathrm{hi}}=20$ (curve 1) and 40 (curve 2). For the dashed curves, $v_{\text {hi }}=0$, and $v_{\text {he }}=0.2$ (curve 3 ) and 0.5 (curve 4 ). The soliton solutions do not exist for $T_{\mathrm{he}} / T_{\mathrm{hi}} \leq 0.1$.

in cold ion density ratio $N_{\text {ci }} / N_{0}$ can lead to decrease in $\phi_{\max }$ for slow ion and ion-acoustic solitons, but has no effect on the $\phi_{\max }$ for the electron-acoustic solitons (not shown). Furthermore, increases in the hot electron and hot ion beam velocities lead to decreases in $\phi_{0}$ for a fixed Mach number but have negligible effect on $\phi_{\max }$ as compared to that of a no beam case (not shown).

In Fig. 5, we have shown the profiles for the potential, $\phi$, of the electron-acoustic solitons for different values of normalized hot electron and ion beam speeds for the case of $N_{\mathrm{ce}} / N_{0}=0.5, N_{\mathrm{ci}} / N_{0}=0.3, T_{\mathrm{ce}} / T_{\mathrm{hi}}=T_{\mathrm{ci}} / T_{\mathrm{hi}}=0.01$, $T_{\mathrm{he}} / T_{\mathrm{hi}}=1.0$, and $M=53.429$. It is seen that both the amplitude and the width of the electron-acoustic solitons decrease with the increase of hot ion beam speed (cf. solid curves) and hot electron beam speed (cf. dashed curves). Further, both amplitude and width decrease with the decrease of $T_{\mathrm{he}} / T_{\mathrm{hi}}$ and the electron-acoustic soliton solutions do not exist for $T_{\text {he }} / T_{\text {hi }} \leq 0.1$ (not shown). From Fig. 5 , it is seen that the maximum value of the potential, $\phi_{\max }=(0.1-0.226)$, and the associated soliton width, $W$, defined as full width at half maximum is $W=(31-44)$. The electric field associated with the solitary potential profile will have a bipolar structure as can be verified by differentiating the profiles.

In Fig. 6, we have shown the profiles for the potential, $\phi$, of the ion-acoustic solitons for the plasma parameters: $N_{\mathrm{ce}} / N_{0}=0.5, N_{\mathrm{ci}} / N_{0}=0.3, T_{\mathrm{ce}} / T_{\mathrm{hi}}=T_{\mathrm{ci}} / T_{\mathrm{hi}}=0.01, M=1.746$,

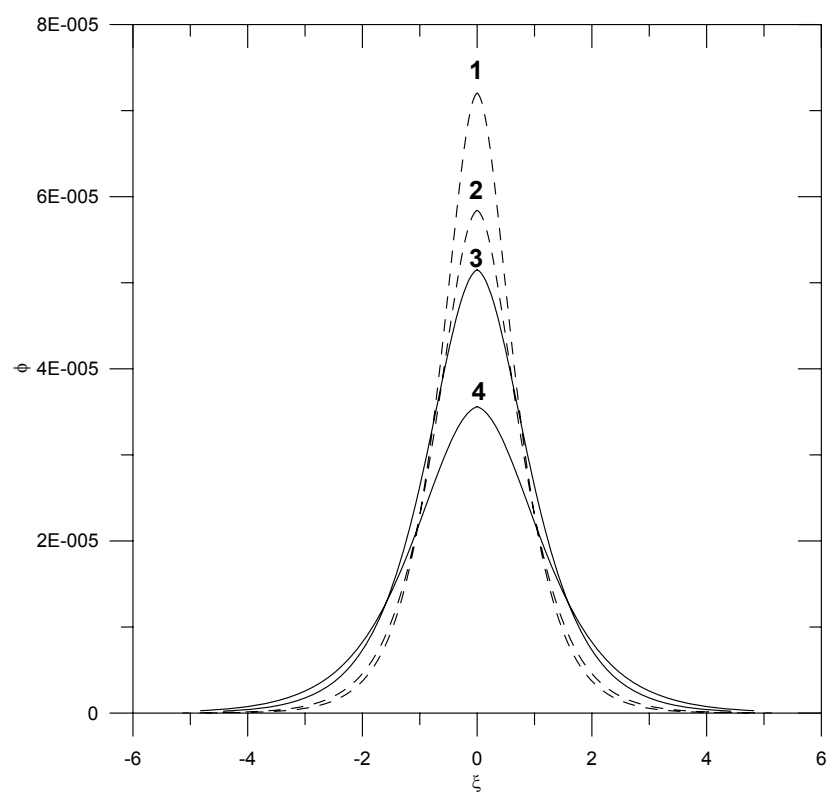

Fig. 6. Shows variation of the ion-acoustic potential $\phi$ versus $\xi$ for $N_{\text {ce }} / N_{0}=0.5, N_{\text {ci }} / N_{0}=0.3, T_{\text {ce }} / T_{\mathrm{hi}}=T_{\text {ci }} / T_{\mathrm{hi}}=0.01, v_{\mathrm{he}}=0$. The Mach number is $M=1.746$ for all the curves. For dashed curves, $T_{\mathrm{he}} / T_{\mathrm{hi}}=0.1$, and $v_{\mathrm{hi}}=0.001$ (curve 1) and 0.0015 (curve 2). For the solid curves, $T_{\mathrm{he}} / T_{\mathrm{hi}}=1.0$, and $v_{\mathrm{he}}=0.001$ (curve 3 ) and 0.0015 (curve 4).

$v_{\text {he }}=0$, and $v_{\text {hi }}=0.001$ and 0.0015 . The dashed and solid curves are for $T_{\mathrm{he}} / T_{\mathrm{hi}}=0.1$ and 1.0 , respectively. In this case, the maximum soliton amplitudes decrease with the increase of $T_{\text {he }} / T_{i}$ and $v_{\text {hi }}$ (cf. dashed and solid curves), though slightly. From the solid and dashed curves, it is noticed that the maximum potential $\phi_{\max }$ varies from 0.00003 to 0.00007 and soliton width $W$ varies from 1.9 to 2.2. Variation of $v_{\text {he }}$ has no effect on the ion-acoustic solitons.

Fig. 7 shows the profiles for the potential, $\phi$, of the slow ion-acoustic solitons for the same plasma parameters as in Figure 6 but for $M=0.22$. In this case also, the maximum soliton amplitudes decrease with the increase of $T_{\text {he }} / T_{\text {hi }}$ (cf. dashed and solid curves). However, the soliton width $\mathrm{W}$ increases with an increase in $T_{\mathrm{he}} / T_{\mathrm{hi}}$. The maximum potential $\phi_{\max }=0.0006$ and 0.00024 , and $W=2$ and 4 , respectively, for $T_{\mathrm{he}} / T_{\mathrm{hi}}=0.1$ and 1.0. Variations of $v_{\mathrm{he}}$ and $v_{\mathrm{hi}}$ have no effect on the slow ion-acoustic solitons.

\section{Discussion and application to plasma sheet boundary layer}

We have described a general analysis for studying largeamplitude ion- and electron-acoustic solitary waves in an unmagnetized four-component plasma system consisting of two, cold and hot, electron populations and two types of, cold 


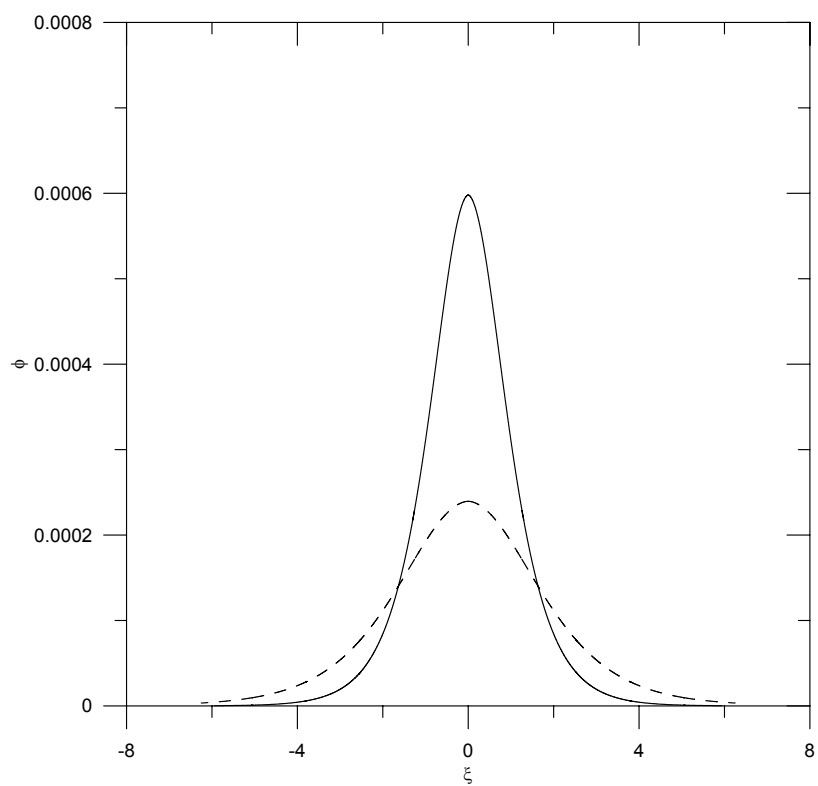

Fig. 7. Shows variation of the slow ion-acoustic potential $\phi$ versus $\xi$ for $N_{\text {ce }} / N_{0}=0.5, N_{\text {ci }} / N_{0}=0.3, T_{\text {ce }} / T_{\mathrm{hi}}=T_{\text {ci }} / T_{\text {hi }}=0.01, v_{\text {he }}=0$, $v_{\text {hi }}=0.0$ and $M=0.22$. Here, the dashed and solid curves are for $T_{\mathrm{he}} / T_{\mathrm{hi}}=1.0$ and 0.1 , respectively. Variations of hot electron and hot ion beam speeds have no effect on the amplitude as well as soliton width.

and hot, ion populations. The model treats all the species as mobile, and both the hot species can have an arbitrary beam velocity.

The most important results of the present model is that the introduction of the cold ion species gives rise to a new mode, the slow ion-acoustic mode, at low Mach numbers, in addition to the ion- and electron-acoustic modes which can exist in a three-component plasma system (Lakhina et al., 2008). For the parameters considered in this paper, electron-acoustic ESWs can have either negative or positive potential depending on $R$, the fractional number density of the cold electrons relative to that of the ions (or total electrons) number density, where as both, slow ion-acoustic and ion-acoustic ESWs can have only positive potential structures. Another important result is that for a fixed Mach number, increases in the beam speeds of either hot electrons or hot ions can lead to reduction in the amplitudes of the ion-and electron-acoustic solitons. The amplitudes of slow ion-acoustic modes remain unaffected by any change in the beam speed of hot electrons or hot ions.

The spacecraft observations (Parks et al., 1984, 1998; Onsager et al., 1993; Takahashi and Hones, 1988) in the Earth's plasma sheet boundary layer show the existence of cold and hot electrons (or some times electron beams) having energies of the order of a few $\mathrm{eV}$ to a few $\mathrm{keV}$, respectively, and background cold ions and warm ions/or ion beams with ener- gies from a few $\mathrm{keV}$ to tens of $\mathrm{keV}$. The properties of plasma components of the plasma sheet boundary layer region are summarized (Grabbe and Eastman, 1984) below:

1. Ion beams have energies $\sim 1$ to $>40 \mathrm{keV}$ with densities $N_{\text {hi }} \sim 0.1$ to $0.8 N_{0}$, where $N_{0}$ is the total (electron or ion) plasma density, and thermal velocities $C_{\mathrm{hi}} \sim 0.1 v_{\mathrm{hi}}$, where $v_{\mathrm{hi}}$ is the ion beam velocity. Typically $N_{0}=0.5 \mathrm{~cm}^{-3}$ which corresponds to the electron plasma frequency $f_{p e} \sim 0.63 \times 10^{4} \mathrm{~Hz}$.

2. Low-energy ions of density $N_{\mathrm{ci}} \sim 0.1-0.8 N_{0}$ and temperatures $T_{\mathrm{ci}} \sim 50-100 \mathrm{eV}$.

3. Hot electrons with temperature $T_{\mathrm{he}} \sim 200-500 \mathrm{eV}$ and densities $N_{\mathrm{he}} \sim 0.5-1.0 N_{0}$, as determined by averaging electron spectra above the photoelectron threshold.

4. There is a high possibility of a cold electron component having densities $N_{\mathrm{ce}} \sim 0.0-0.5 N_{0}$, and temperatures $T_{\mathrm{ce}} \sim 1 \mathrm{eV}$ to a few $10 \mathrm{~s} \mathrm{eV}$.

In view of the above, we choose the plasma parameters as: $N_{\mathrm{ci}} / N_{0}=0.3, N_{\mathrm{hi}} / N_{0}=0.7, N_{\mathrm{ce}} / N_{0}=N_{\mathrm{he}} / N_{0}=0.5$, $T_{\mathrm{hi}}=500 \mathrm{eV}, \quad v_{\mathrm{hi}} / C_{\mathrm{hi}}=10-20, T_{\mathrm{ce}} / T_{\mathrm{hi}}=0.01, \quad T_{\mathrm{ci}} / T_{\mathrm{hi}}=0.01-$ 0.1 , and $T_{\mathrm{he}} / T_{\mathrm{hi}}=1.0$. Then, from Figs. 5-7, we note that the typical potential amplitude, $\phi_{\max }$, and the widths, $W$, for the slow ion-acoustic, ion-acoustic and electron-acoustic solitons are $\phi_{\max } \sim(0.00024-0.0006)$ and $W \sim(2-4), \phi_{\max } \sim(0.00003-0.00007)$ and $W \sim(1.9-2.2)$, and $\phi_{\max } \sim(0.1-0.226)$ and $W \sim(31-44)$, respectively. It is interesting to note that the electric fields associated with these solitons would have bipolar structures with maximum amplitudes, $E_{\max } \approx\left(T_{\mathrm{hi}} \phi_{\max } / e \lambda_{d i} W\right)$. For the case of $R=0.5, T_{\mathrm{ce}}=1 \mathrm{eV}, T_{\mathrm{hi}}=500 \mathrm{eV}$ and $T_{\mathrm{he}}=(50-500) \mathrm{eV}$ and $N_{0}=0.5 \mathrm{~cm}^{-3}, \lambda_{\mathrm{di}}=234 \mathrm{~m}$, we find $E_{\max } \sim(0.1-0.5) \mathrm{mV} / \mathrm{m}$, $(0.03-0.07) \mathrm{mV} / \mathrm{m}$, and $(4-10) \mathrm{mV} / \mathrm{m}$ for the slow ionacoustic, ion-acoustic and electron-acoustic solitons, respectively. All the three types of solitons are positive potential structures in this case and the associated electric fields would be bipolar. The widths of these ESWs would be $\sim(400$ 900) $\mathrm{m},(400-500) \mathrm{m}$ and $(7-10) \mathrm{km}$, respectively.

We would like to point out that our model deals with the nonlinear state of the system. It cannot claim that the modes arise from the linear instability as happens in the simulations of Lu et al. (2005) where an electron beam streaming at $\sim 10$ cold electron thermal speeds or more drives the linear modes which saturates by trapping the electrons. In our case, the solitary structures are the nonlinear modes of the system, just as the BGK modes are the nonlinear modes of the VlasovPoisson system. Since we treat each plasma component as fluid, this naturally restrict the electrostatic structures having scale lengths greater than the Debye length. 
Acknowledgements. GSL thanks the Indian National Science Academy, New Delhi, India, for the support under the Senior Scientist scheme. FV thanks the Fonds voor Wetenschappelijk Onderzoek (Vlaanderen) for a research grant.

Edited by: P.-L. Sulem

Reviewed by: three anonymous referees

\section{References}

Ashour-Abdalla, M. and Okuda, H.: Electron acoustic instabilities in the geomagnetic tails, Geophys. Res. Lett., 13, 366-369, 1986.

Backrud-Ivgren, M., Stenberg, G., André, M., Morooka, M., Hobara, Y., Joko, S., Rönnmark, K. Cornilleau-Wehrlin, N., Fazakerley, A., and R'eme, H., Cluster observations and theoretical identification of broadband waves in the auroral region, Ann. Geophy., 23, 3739-3752, 2005.

Bale, S. D., Kellogg, P. J., Larson, D. E., Lin, R. P., Goetz, K., and Lepping, R. P.: Bipolar electrostatic structures in the shock transition region: Evidence of electron phase space holes, Geophys. Res. Lett., 25, 2929-2932, 1998.

Bernstein I. B., Greene J. M., and Kruskal M. D.: Exact Nonlinear Plasma Oscillations, Physical Review, 108, 546-550, 1957.

Berthomier, M., Pottelette, R., and Malingre, M.: Solitary waves and weak double layers in a two-electron temperature auroral plasma, J. Geophys. Res., 103, 4261-4270, 1998.

Berthomier, M., Pottelette, R., Malingre, M., and Khotyaintsev, Y.: Electron acoustic solitons in an electron-beam plasma system, Phys. Plasmas 7, 2987-2994, 2000.

Berthomier, M., Pottelette, R., Muschietti, L., Roth, I., and Carlson, C. W.: Scaling of 3-D solitary waves observed by FAST and POLAR, Geophys. Res. Lett, 30, 2148, doi:10.1029/2003GL018491, 2003.

Bharuthram, R. and Shukla, P. K., Multi-dimensional Solitons and Double Layers in a Magnetized Plasma, Physica Scripta, 34, 732-735, 1985.

Boström, R., Gustafsson, G., Hollback, B., Holmgren, G., Koskinen, H., et al.: Characteristics of solitary waves and double layers in the magnetospheric plasma, Phys. Rev. Lett., 61, 82-85, 1988.

Bounds, S. R., Pfaff, R. F., Knowlton, S. F., Mozer, F. S., Temerin, M. A., and Kletzing, C. A.: Solitary potential structures associated with ion and electron beams near $1 R_{E}$ altitude, J. Geophys. Res., 104, 28709, doi:10.1029/1999JA900284, 1999.

Buti, B.: Ion-acoustic holes in a two-electron-temperature plasma, Phys. Lett. A., 76, 251-254, 1980.

Cattaert, T., Verheest, F., and Hellberg, M. A.: Potential hill electron-acoustic solitons and double layers in plasmas with two electron species, Phys. Plasmas., 12, 042901, doi:10.1063/1.1868733, 2005.

Cattell, C. A., Dombeck, J., Wygant, J. R., Hudson, M. K., Mozer, F. S., Temerin, M. A., Peterson, W. K., Kletzing, C. A., Russell, C. T., and Pfaff, R. F.: Comparisons of Polar satellite observations of solitary wave velocities in the plasma sheet boundary and the high altitude cusp to those in the auroral zone, Geophys. Res. Lett., 26, 425-428, 1999.
Chen, L. J., Pickett, J. s., Kinter, P., Franz, J., and Gurnett, D. A.: On the width-amplitude inequality of electron phase space holes, J. Geophys. Res., 110 , A09211, doi:10.1029/2005JA011087, 2005.

Dubouloz, N., Pottelette, R., Malingre, M., and Treumann, R. A.: Generation of broadband electrostatic noise by electron acoustic solitons, Geophys. Res. Lett., 18, 155-158, 1991.

Dubouloz, N., Treumann, R. A., Pottelette, R., and Malingre, M.: Turbulence generated by a gas of electron acoustic solitons, J. Geophys. Res., 98, 17 415-17 422, 1993.

El-Taibany, W. F.: Electron-acoustic solitary waves and double layers with an electron beam and phase space electron vortices in space plasmas, J. Geophys. Res., 110, A01213, doi:10.1029/2004JA010525, 2005.

Ergun, R. E., Carlson, C. W., McFadden, J. P., et al.: FAST Satellite Observations of Large-Amplitude Solitary Structures, Geophys. Res. Lett., 25, 2041-2044, 1998.

Franz, J. R., Kintner, P. M., and Pickett, J. S.: POLAR observations of coherent electric field structures, Geophys. Res. Lett., 25, 1277-1280, 1998.

Gary, S. P. and Tokar, R. L. The electron-acoustic mode, Phys. Fluids, 28, 2439-1441, 1985

Goldman, M. V., Oppenheim, M. M., and Newman, D. L.: Nonlinear Two-Stream Instabilities as an explanation for the auroral bipolar wave structures, Geophys. Res. Lett., 26, 1821-1824, 1999.

Ghosh, S. S., Pickett, J. S., Lakhina, G. S., Winningham, J. D., Lavraud, B. P. M. E., Décréau, B.: Parametric Analysis of Positive Amplitude Electron Acoustic Solitary Waves in a Magnetized Plasma and its Application to Boundary Layers, J. Geophys. Res., 113, A06218, doi:10.1029/2007JA012768, 2008.

Grabbe, C. L. and Eastman, T. E.: Generation of broadband electrostatic noise by ion beam instabilities in the magnetotail, J. Geophys. Res., 89, 3865-3872, 1984.

Hudson, M. K., Lotko, W., Roth, I., and Witt, E.: Solitary waves and double layers on auroral field lines, J. Geophys. Res., 88, 916, doi:10.1029/JA088iA02p00916, 1983.

Jovanovic, D. and Shukla, P. K.: Nonlinear Model for Coherent Electric Field Structures in the Magnetosphere, Phys. Rev. Lett., 84, 4373-4376, 2000.

Kakad, A. P., Singh, S. V., Reddy, R. V., Lakhina, G. S., Tagare, S. G., and Verheest, F.: Generation mechanism for electron acoustic solitary waves, Phys. Plasmas., 14, 052305, doi:10.1063/1.2732176, 2007.

Kojima, H., Matsumoto, H., Chikuba, S., Horiyama, S., Ashour-Abdalla, M., and Anderson, R. R.: Geotail Waveform Observations of Broadband/narrowband Electrostatic Noise in the Distant Tail, J. Geophys. Res., 102, 14439-14455, doi:10.1029/97JA00684, 1997.

Koskinen, H. E. J., Lundin, R., and Holback, B.: On the plasma environment of solitary waves and weak double layers, J. Geophys. Res., 95, 5921-5929, 1990.

Lakhina, G. S., Tsurutani, B. T., Kojima, H., Matsumoto, H.: "Broadband" plasma waves in the boundary layers, J. Geophys. Res. 105, 27 791-27 831, 2000.

Lakhina, G. S., Tsurutani, B. T., and Pickett, J.: Association of Alfvén waves and proton cyclotron waves with electrostatic bipolar pulses: magnetic hole events observed by Polar, Nonlin. Processes Geophys., 11, 205-213, 2004, 
http://www.nonlin-processes-geophys.net/11/205/2004/.

Lakhina, G. S., Kakad, A. P., Singh, S. V. and Verheest, F.: Ionand electron-acoustic solitons in two-electron temperature space plasmas, Phys. Plasmas., 15, 062903, doi:10.1063/1.2930469, 2008.

Lin, C. S., Burch, J. L., Shawhan, S. D. and Gurnett, D. A.: Correlation Of Auroral hiss and upward electron beams near the polar Cusp, J. Geophys. Res., 89, 935, doi:10.1029/JA089iA02p00925, 1984.

Lu, Q., Wang, S., and Dou, X., Electrostatic waves in an electron-beam plasma system, Phys. Plasmas, 12, 072903, doi:10.1063/1.1951367, 2005.

Mace, R. L. and Hellberg, M. A.: Higher order electron longitudinal modes in a two electron temperature plasma, J. Plasma Phys., 43, 239-247, 1990.

Mace, R. L. and Hellberg, M. A.: The Korteweg-de VriesZakharov-Kuznetsov equation for electron-acoustic waves, Phys. Plasmas., 8, 2649-2656, 2001.

Matsumoto, H., Kojima, H., Miyatake, T., Omura, Y., Okada, M., Nagano, I., and Tsutui, M.: Electrostatic Solitary Waves (ESW) in the Magnetotail: BEN Wave Forms Observed by GEOTAIL, Geophys. Res. Lett., 21, 2915-2918, 1994.

McKenzie, J. F., Dubinin, E., Sauer, K., and Doyle, T. B.: The application of the constants of the motion to nonlinear stationary waves in complex plasmas: a unified fluid dynamic point of view, J. Plasma Phys., 70, 431-462, 2004.

Muschietti, L., Ergun, R. E., Roth, I., and Carlson, C. W.: PhaseSpace Electron Holes along Magnetic Field Lines, Geophys. Res. Lett., 26, 1093-1096, 1999.

Omura, Y., Matsumoto, H., Miyake, T., and Kojima, H.: Electron beam instabilities as generation mechanism of electrostatic solitary waves in the magnetotail, J. Geophys. Res., 101, 2685-2697, 1996.

Onsager, T. G., Thomsen, M. F., Elphic, R. C., Gosling, J. T., Anderson, R. R., and Kettmann, G.: Electron Generation of Electrostatic Waves in the Plasma Sheet Boundary Layer, J. Geophys. Res., 98, 15 509-15 519, 1993.

Parks, G. K., McCarthy, M., Fitzenreiter, R. J., Etcheto, J., Anderson, K . A., Anderson R. R., ANDERSO5N , Eastman, T. E., Frank, L. A., Gurnett, D. A., Huang, C ., Lin, R. P., Lui, A. T. Y., Ogilvie, K. W., Pedersen, A., Reme, H., and Williams, D . J.: Particle and field characteristics of the high-latitude plasma sheet boundary layer, J. Geophys. Res., 89, 8885-8906, 1984.

Parks, G., Chen, L. J., McCarthy, M., Larson, D., Lin, R. P., Phan, T., Reme, H., and Sanderson, T.: New observations of ion beams at the outer edge of the plasma sheet boundary layer, Geophys. Res. Lett., 25, 3285-3288, 1998.

Pickett, J. S., Menietti, J. D., Gurnett, D. A., Tsurutani, B., Kintner, P. M., Klatt, E., and Balogh, A.: Solitary potential structures observed in the magnetosheath by the Cluster spacecraft, Nonlin. Processes Geophys., 10, 3-11, 2003,

http://www.nonlin-processes-geophys.net/10/3/2003/.

Pickett, J. S., Chen, L.-J., Kahler, S. W., Santolík, O., Goldstein, M. L., Lavraud, B., Décréau, P. M. E., Kessel, R., Lucek, E., Lakhina, G. S., Tsurutani, B. T., Gurnett, D. A., CornilleauWehrlin, N., Fazakerley, A., Rème, H., and Balogh, A.: On the generation of solitary waves observed by Cluster in the nearEarth magnetosheath, Nonlin. Processes Geophys., 12, 181-193, 2005 , http://www.nonlin-processes-geophys.net/12/181/2005/.

Pottelette, R., Malingre, M., Dubouloz, N., Aparicio, B., Lundin, R., Holmgren, G., and Marklund, G.: High Frequency Waves in the Cusp/cleft Regions, J. Geophys. Res., 95, 5957-5971, 1990.

Pottelette, R., Ergun, R. E., Treumann, R. A., Berthomier, M., Carlson, C. W., McFadden, J. P., and Roth, I.: Modulated electronsacoustic waves in auroral density waves in auroral density cavities' FAST observations, Geophys. Res. Lett., 26, 2629-2632, 1999.

Qian, S., Lotko, W. and Hudson, M. K.: Dynamics of localized ion-acoustic waves in a magnetized plasma, Phys. Fluids., 31, 2190-2200, 1988.

Reddy, R. V. and Lakhina, G. S.: Ion-acoustic double layers and solitons in auroral plasma, Planet. Space Sci., 39, 1343-1350, 1991.

Reddy, R. V., Lakhina, G. S. and Verheest, F.: Ion-acoustic double layers and solitons in multispecies auroral beam plasmas, Planet. Space Sci., 40, 1055-1062, 1992.

Singh, S. V., Reddy, R. V., and Lakhina, G. S.: Broadband Electrostatic Noise due to Nonlinear Electron Acoustic Waves, Adv. Space Res., 28(11), 1643-1648, 2001.

Singh S. V. and Lakhina G. S.: Generation of Electron-Acoustic Waves in the Magnetosphere, Planet. Space Sci., 49, 107-114, 2001.

Singh, S. V. and Lakhina, G. S.: Electron acoustic solitary waves with non-thermal distribution of electrons., Nonlin. Proc. Geophys., 11, 275-279, 2004.

Tagare, S. G., Singh, S. V., Reddy, R. V., and Lakhina, G. S.: Electron acoustic solitons in the Earth's magnetotail, Nonlinear Proc. Geophys., 11, 215-218, 2004

Temerin, M., Cerny, K., Lotko, W., and Mozer, F. S.: Observations of double layers and solitary waves in the auroral plasma, Phys Rev. Lett., 48, 1175-1179, 1982.

Takahashi, K. and Hones Jr., E. W.: ISEE 1 and 2 observations of ion distributions at the plasma sheet-tail lobe boundary, J. Geophys. Res., 93, 8558-8582, 1988

Teste, A., Fontaine, D., Sauvaud, J.-A., Maggiolo, R., Canu, P., and Fazakerley, A.: CLUSTER observations of electron outflowing beams carrying downward currents above the polar cap by northward IMF, Ann. Geophys., 25, 953-969, 2007, http://www.ann-geophys.net/25/953/2007/.

Tokar, R. L. and Gary, S. P.: Electrostatic hiss and the beam driven electron-acoustic instability in the dayside polar cusp, Geophys. Res. Lett., 11, 1180-1183, 1984

Tsurutani, B. T., Arballo J. K., Lakhina G. S., Ho, C. M., Buti, B., Pickett, J. S., and Gurnett, D. A.: Plasma Waves in the Dayside Polar Cap Boundary Layer : Bipolar and Monopolar Electric Pulses and Whistler Mode Waves, Geophys. Res. Lett., 25, 4117-4120, 1998

Verheest, F., Cattaert, T., Lakhina, G. S., and Singh, S. V.: Gasdynamic description of electrostatic solitons, J. Plasma Phys., 70, 237-250, 2004.

Verheest, F., Cattaert, T., and Hellberg, M.: Compressive and Rarefactive Electron-Acoustic Solitons and Double Layers in Space Plasmas, Space Sci. Rev., 121, 299-311, 2005.

Verheest, F., Hellberg, M. A., and Lakhina, G. S., Necessary conditions for the generation of acoustic solitons in magnetospheric and space plasmas with hot ions, Astrophys. Space Sci. Trans., $3,15-20,2007$ 
Vogiatzis I. I., Fritz, T. A., Zong, Q.-G., and Sarris, E. T.: Two distinct energetic electron populations of different origin in the Earths magnetotail: a Cluster case study, Ann. Geophys., 24, 1931-1948, 2006,

http://www.ann-geophys.net/24/1931/2006/.
Watanabe, K. and Tanuti, T.: Electron-Acoustic Mode in a Plasma of Two-Temperature Electrons, J. Phys. Soc. Japan, 4, 1819$1820,1977$. 